\title{
Finite Element Simulation of Nanostructuring Burnishing
}

\author{
V. P. Kuznetsov ${ }^{a}$, I. Yu. Smolin*b,c, A. I. Dmitriev ${ }^{b, c}$, D. A. Konovalov ${ }^{d}$, \\ A. V. Makarov ${ }^{d}$, A. E. Kiryakov ${ }^{a}$, and A. S. Yurovskikh ${ }^{e}$ \\ ${ }^{a}$ Kurgan State University, Kurgan, 640669 Russia \\ ${ }^{b}$ Institute of Strength Physics and Materials Science, Siberian Branch, Russian Academy of Sciences, \\ Tomsk, 634021 Russia \\ ${ }^{c}$ National Research Tomsk State University, Tomsk, 634050 Russia \\ d Institute of Machine Science, Ural Branch, Russian Academy of Sciences, Ekaterinburg, 620219 Russia \\ ${ }^{e}$ Ural Federal University, Ekaterinburg, 620002 Russia \\ *smolin@ispms.tsc.ru
}

Received June 8, 2011

\begin{abstract}
Dynamics simulation of burnishing of a thin steel layer beneath an indenter applied with a constant force and then moving with a constant velocity was performed by the finite element method in the plane strain approximation. The indenter was modeled by a perfectly rigid body, and the steel was modeled by an elastoplastic body with isotropic hardening according to an experimentally defined law. The regularities of changes in the stressstrain state of the material near the treated surface were studied and mechanisms of the formation of a nanostructured layer were disclosed. The effect of the friction coefficient and the burnishing force on the height of a bulge of edged material was analyzed. The results of studies agree well with experimental data.
\end{abstract}

DOI: $10.1134 / \mathrm{S} 1029959913010074$

Keywords: nanostructuring burnishing, surface layer, finite element simulation, cyclic alternate action.

\section{INTRODUCTION}

Surface burnishing on modern multiprocessing machines is a highly efficient method of surface finish of high-precision parts [1-18]. Spherical [5, 6, 10-13] or cylindrical diamond burnishing [14-17] involves plastic deformation and structural changes in a surface layer, thus providing improvement of qualitative and service surface properties such as roughness, hardness, wear resistance, and fatigue strength [5-9]. Diamond burnishing, which uses indenters with a working section made of natural or synthetic diamonds, features a low friction coefficient; therefore, in studies with diamond burnishing, the frictional force at the "indenter - treated surface" contact is normally neglected.

A considerable increase in hardness, wear resistance, and fatigue strength of structural steel surfaces can be attained by nanostructuring burnishing in which indenters with increased friction coefficients and thermal stability ensure a high shear strain level [6]. Nanostructuring burnishing forms an enlarged bulge of plastically edged metal which is a stress microconcentrator assisting structural dispersion of a thin surface layer. However, the growth of the bulge can lead to destabilization of the dynamic burnishing system and initiate self-vibration [4, 18]. Thus, nanostructuring surface burnishing of structural steels requires theoretical substantiation of its modes to control plastic strain accumulation without impairing the dynamic stability of the process. Another important problem is to clarify the effect of the ratio between the burnishing force and the friction coefficient of the indenter material on the character of deformation processes.

Note that the existing theoretical models [19-21], which are based on analytical description, and the numerical models that use static problem statements fail to reproduce in full measure the actual pattern of the processes occurring in a thin surface layer in burnishing, the more so in nanostructuring burnishing. This is due to both dynamics of the process and extremely complex nonuniform stress-strain distribution in the thus loaded material. Similar calculations are most often taken in various approximations which give data on the process of interest but only for a particular case. So the coordinate grid method used for theoretical study of rolling and bur- 
nishing [22] makes it possible to analyze, in the context of solid mechanics and plastic working, the stress-strain state of isotropic material at the deformation site (at the "indenter - burnished surface" contact zone). A more preferable way is to use numerical simulation methods in which a system of differential equations of solid mechanics is integrated with regard for boundary conditions and elastoplastic properties of a surface layer of test material.

The main method of numerical research in stress and strain distribution remains to be the finite element method. Until recently, the basic formation mechanisms of a surface layer in machine parts hardened by severe plastic deformation have been considered in static and quasistatic problem statements. The authors of [22-27] studied roller burnishing and frictional treatment of a surface layer by the finite element method for the conditions of quasistatic deformation with a constant indenter penetration depth. Note that this problem statement is not quite accurate to fit in the main conditions of burnishing. By and large, analysis of the available papers discloses that it is not entirely clear to what extent the main burnishing parameters affect the formation of a specific stressstrain state of material near its treated surface and the structure of a near-surface layer of structural steels which determines functionality of the layer.

The objective of the work was to study the process of nanostructuring burnishing and the peculiarities of the stress-strain state of a surface layer of the thus treated material by the dynamic variant of the finite element method. The simulation was performed for the process in dynamics and with a specified constant indenter pressing force. This is the main difference of the method from conventional approaches used to study the stress-strain state of a near-surface layer. The primary task was to determine the peculiarities of the stress-strain state capable of providing the formation mechanisms of a nanostructured surface layer - friction-induced shear and rotational deformation $[28,29]$. Other tasks to be solved were the following:

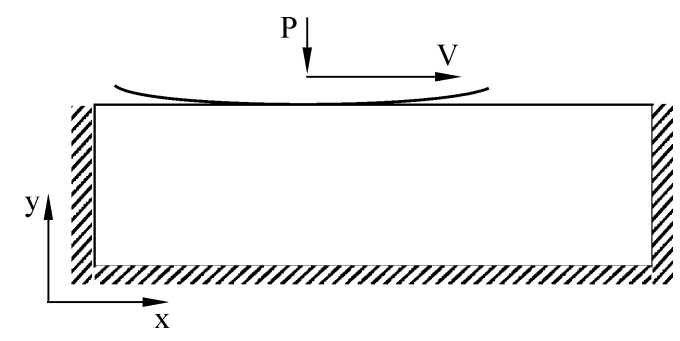

Fig. 1. Schematic diagram of the model specimen and loading conditions

PHYSICAL MESOMECHANICS Vol.16 No. 12013
1) to study the peculiarities of the stress-strain state of material at the stage of penetration of an indenter and in its motion in one and several passes;

2) to analyze the effect of the burnishing force and the friction coefficient on the height of a bulge of edged material, and

3) to estimate the accumulated plastic strain in a surface layer depending on the number of passes.

\section{GEOMETRIC MODEL. BOUNDARY CONDITIONS}

We simulated burnishing of a steel specimen in the two-dimensional statement for the plane stress state. The calculations were performed by the ANSYS/LS-DYNA finite element program. The loading was realized in two stages and was specified through calculating the interaction between the upper face of the deformed specimen and the indenter which was an absolutely rigid body with its boundary being part of a sphere of radius $R=2 \mathrm{~mm}$. First, the indenter was pressed into the specimen with a constant force $P$, whereupon it moved under this force with a constant velocity $V=10 \mathrm{~m} / \mathrm{min}$. Schematic of the model specimen and loading conditions is shown in Fig 1. At the top of the rectangle, part of the indenter is depicted with indication of the burnishing force $P$ and indenter velocity $V$. The rectangle framed on three sides corresponds to part of the treated specimen with boundary conditions of "nonpercolation" or symmetry (displacements perpendicular to the boundaries are prohibited). The dimensions of the domain were $4792 \times$ $1995 \mu \mathrm{m}$.

The parameters varied in the calculations were the friction coefficient and the burnishing force. In one calculation, the sliding friction coefficient $\mu$ and the burnishing force $P$ were fixed. The initial specimen surface was taken to be perfectly smooth.

\section{FINITE ELEMENT GRID}

We chose a grid of four-node quadrilateral elements (simplexes) with linear interpolation of the desired quantities. The grid was crowded from the bottom upwards to provide sufficient details of the grid at the contact surface (Fig. 2) and was horizontally rarefied at the computational domain boundaries to which, by the problem statement, the indenter center fails to come (Fig. 2) and where an abrupt change in the stress-strain state was not expected. Thus, at the initial point in time, the elements at the top of the loaded domain approximated squares and those at the boundaries and at the bottom were rectangles. The 


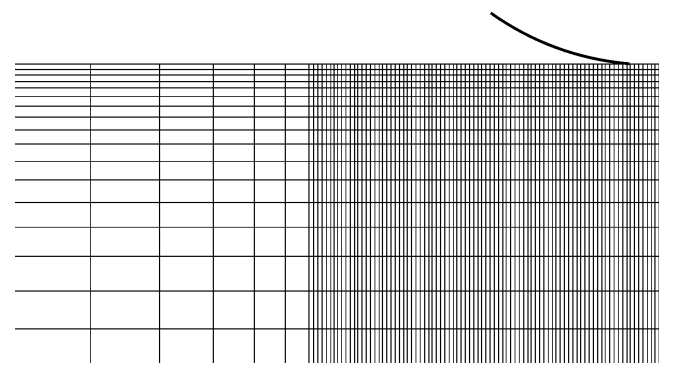

Fig. 2. Fragment of the computation grid near the indentersurface contact point.

number of nodes in the computational grid reached 53352; the number of elements was 51601.

\section{MATHEMATICAL MODEL AND CONSTITUTIVE (PHYSICAL) RELATIONS}

The mechanical characteristics of the specimen were taken to correspond to steel 20X (Russian designation): Young's modulus $E=218 \mathrm{GPa}$, Poisson's ratio $v=0.267$, and density $\rho=7670 \mathrm{~kg} / \mathrm{m}^{3}$. The mechanical behavior was described by an elastoplastic model with the von Mises yield criterion and isotropic hardening. Because the real process involves cooling of the specimen surface and indenter, the heat produced in friction and its effect on the strength characteristics of the steel were ignored.

Numerical simulation of the behavior of the treated material was performed taking into account geometric and physical nonlinearity, i.e., the possibility of large displacements, rotations, and strains for deformation and plastic strains for mechanical response. In this case, the problem is solved in terms of increments for both quasistatic indentation and indenter motion with constant velocity. In the dynamic problem, an explicit time integration scheme was used and the time increments were small according to the stability condition of the numerical scheme.

The mathematical problem statement is based on the variational principle of virtual displacements according to which a virtual variation in internal strain energy is bound to be compensated by the same variation in external applied loads:

$$
\int_{V} \sigma_{i j} \delta \varepsilon_{i j} \mathrm{~d} V=\int_{S} f_{i}^{S} \delta u_{i} \mathrm{~d} S+\int_{V} f_{i}^{B} \delta u_{i} \mathrm{~d} V
$$

where $\sigma_{i j}$ are the Cauchy stress tensor components, $\varepsilon_{i j}=\frac{1}{2}\left(\frac{\partial u_{i}}{\partial x_{j}}+\frac{\partial u_{j}}{\partial x_{i}}\right)$ are the strain tensor components, $u_{i}$ are the displacement vector components, $x_{i}$ are the current coordinates, $f_{i}^{S}$ are the surface force vector components, $f_{i}^{B}$ are the bulk (including inertia) force vector components, $V$ is the volume of a deformed solid, and $S$ is the deformed solid surface to which surface forces are applied.

For realization of the variational principle of virtual displacements, stresses are determined in terms of strains. In this case, the constitutive relations are Hooke's law for elastic solids and more complex dependences for elastoplastic solids. As a result, the problem is reduced to a system of equations for displacement increments.

Thus, at each time step, the displacement increment $\mathrm{d} u_{i}$ is determined from the equation of motion (or equilibrium equation in the static case). The displacement increment is used to calculate the total strain increment $\mathrm{d} \varepsilon_{i j}$ which can be expanded into the elastic and plastic strain increments:

$$
\mathrm{d} \varepsilon_{i j}=\mathrm{d} \varepsilon_{i j}^{\mathrm{e}}+\mathrm{d} \varepsilon_{i j}^{\mathrm{pl}} .
$$

The plastic strain increments are determined from the plastic flow rule [20]

$$
\mathrm{d} \varepsilon_{i j}^{\mathrm{pl}}=\mathrm{d} \lambda \frac{\partial f\left(\sigma_{i j}\right)}{\partial \sigma_{i j}}
$$

with the von Mises yield criterion

$$
f\left(\sigma_{i j}\right)=\sigma_{\text {eq }}-\sigma_{\mathrm{s}}\left(\varepsilon_{\mathrm{eq}}^{\mathrm{pl}}\right)=0,
$$

where $\sigma_{\text {eq }}=\sqrt{3 / 2 s_{i j} s_{i j}}$ is the von Mises equivalent stress, $\sigma_{\mathrm{s}}\left(\varepsilon_{\mathrm{eq}}^{\mathrm{pl}}\right)$ is the yield strength dependent on accumulated plastic strains according to the specified hardening curve (Fig. 3), $s_{i j}=\sigma_{i j}+P_{\mathrm{s}} \delta_{i j}$ is the stress tensor deviator, $P_{\mathrm{s}}$ is pressure, and $\delta_{i j}$ is the Kronecker symbol.

The elastic strain increments are determined as a difference of the total and plastic strain increments and then the stress increments are calculated from Hooke's law written in increments:

$$
\mathrm{d}^{J} \sigma_{i j}=D_{i j k l} \mathrm{~d} \varepsilon_{k l}^{\mathrm{e}},
$$

where $\mathrm{d}^{\mathrm{J}} \sigma_{i j}=\mathrm{d} \sigma_{i j}-\sigma_{i k} \mathrm{~d} \omega_{j k}-\sigma_{j k} \mathrm{~d} \omega_{i k}$ is the Jaumann stress increment which takes into account rotation of a solid as a whole, $D_{i j k l}$ is the matrix of elastic coefficients, and $\omega_{i j}=\frac{1}{2}\left(\frac{\partial u_{i}}{\partial x_{j}}-\frac{\partial u_{j}}{\partial x_{i}}\right)$ is the rotation tensor.

The hardening curve was determined in special experiments. The 20X steel specimen was subjected to heat treatment as follows: carburizing (with a carburized layer thickness of $\sim 1.2 \mathrm{~mm}$ ), oil quenching, and tempering at $250{ }^{\circ} \mathrm{C}$ for $2 \mathrm{~h}$. Tests for kinetic indentation were performed on a TEST-MINI-(UT) hardness gage (VNIIAES).

The strain hardening curve was reconstructed from kinetic test results by the procedure described elsewhere 
Table 1. Coefficient $c$ and parameters of approximation of the strain hardening diagram for steel $20 \mathrm{X}$

\begin{tabular}{c|c|c|c|c|c|c}
\hline \multirow{2}{*}{ Metal } & \multicolumn{2}{|c|}{$\begin{array}{c}\text { Coefficient } c \times 10^{-3} \\
\text { for pyramidal } \\
\text { indenters }\end{array}$} & \multicolumn{3}{|c}{$\begin{array}{c}\text { Coefficients } \\
\text { of approximation }\end{array}$} \\
\cline { 2 - 7 } & $90^{\circ}$ & $123^{\circ}$ & $136^{\circ}$ & $\sigma_{0}, \mathrm{MPa}$ & $a_{1}$ & $a_{2}$ \\
\hline Steel 20X & 51 & 114 & 210 & 1200 & 200 & 0.4 \\
\hline
\end{tabular}

[30]. The strain hardening diagram was analytically described as the three-parametric power dependence

$$
\sigma_{\mathrm{s}}=\sigma_{0}\left(1+a_{1} \varepsilon_{\mathrm{eq}}^{\mathrm{pl}}\right)^{a_{2}}
$$

where $\sigma_{0}, a_{1}, a_{2}$ are empirical coefficients and $\sigma_{0}$ has the meaning of stress corresponding to the onset of plastic deformation (yield strength).

The site of loading by pyramidal and conical indenters was described by the parabolic dependence known as Kick's law:

$$
P=c h^{2},
$$

where the coefficient $c$ depends on the indenter angle and elastoplastic properties of indented material and does not depend on the indentation depth.

The tests were performed using three different diamond indenters with vertex angles of $90^{\circ}, 123^{\circ}$, and $136^{\circ}$. Each indenter was used in 10 indentations with recording of indentation diagrams. The coefficients $c$ for formula (2) and the yield strength $\sigma_{0}$ and the coefficients $a_{1}, a_{2}$ for approximation (1) are given in Table 1 . The strain hardening diagram is presented in Fig. 3.

\section{REPRESENTATION OF CALCULATION RESULTS}

The stress-strain state of a surface layer was analyzed with the use of the stress tensor components in the Cartesian coordinate system (Fig. 1), the pressure $P_{\mathrm{s}}$, the von Mises equivalent stresses (stress intensity) $\sigma_{\text {eq }}=$ $\sqrt{3 / 2 s_{i j} s_{i j}}$, and the equivalent accumulated plastic strain tensor (intensity of accumulated plastic strains) $\varepsilon_{\mathrm{eq}}^{\mathrm{pl}}=$

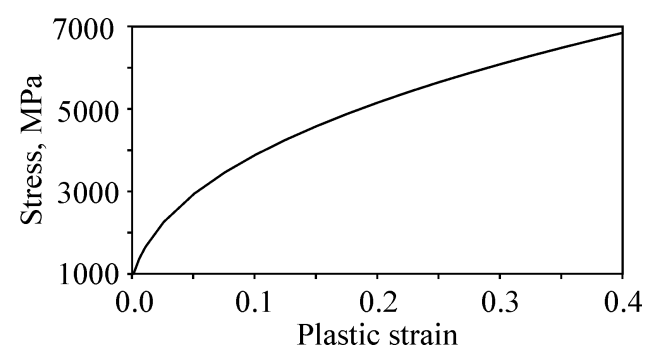

Fig. 3. Strain hardening curve $\sigma_{\mathrm{s}}\left(\varepsilon_{\mathrm{eq}}^{\mathrm{pl}}\right)$. $\int \sqrt{2 / 3 \dot{\varepsilon}_{i j}^{\mathrm{pl}} \dot{\varepsilon}_{i j}^{\mathrm{pl}}} \mathrm{d} t$, where $\dot{\varepsilon}_{\mathrm{eq}}^{\mathrm{pl}}$ are the plastic strain rate tensor components.

\section{CALCULATION RESULTS}

\subsection{Stress and Strain Distribution Beneath an Indenter}

At the first stage, we modeled indentation of the specimen at a constant force in the quasistatic problem statement.

At a friction coefficient of 0.35 and force $P=350 \mathrm{~N}$, the indenter penetration depth was $65 \mu \mathrm{m}$. Figure 4 shows the distributions of pressure, stress intensity, and plastic strain intensity in the specimen beneath the indenter.
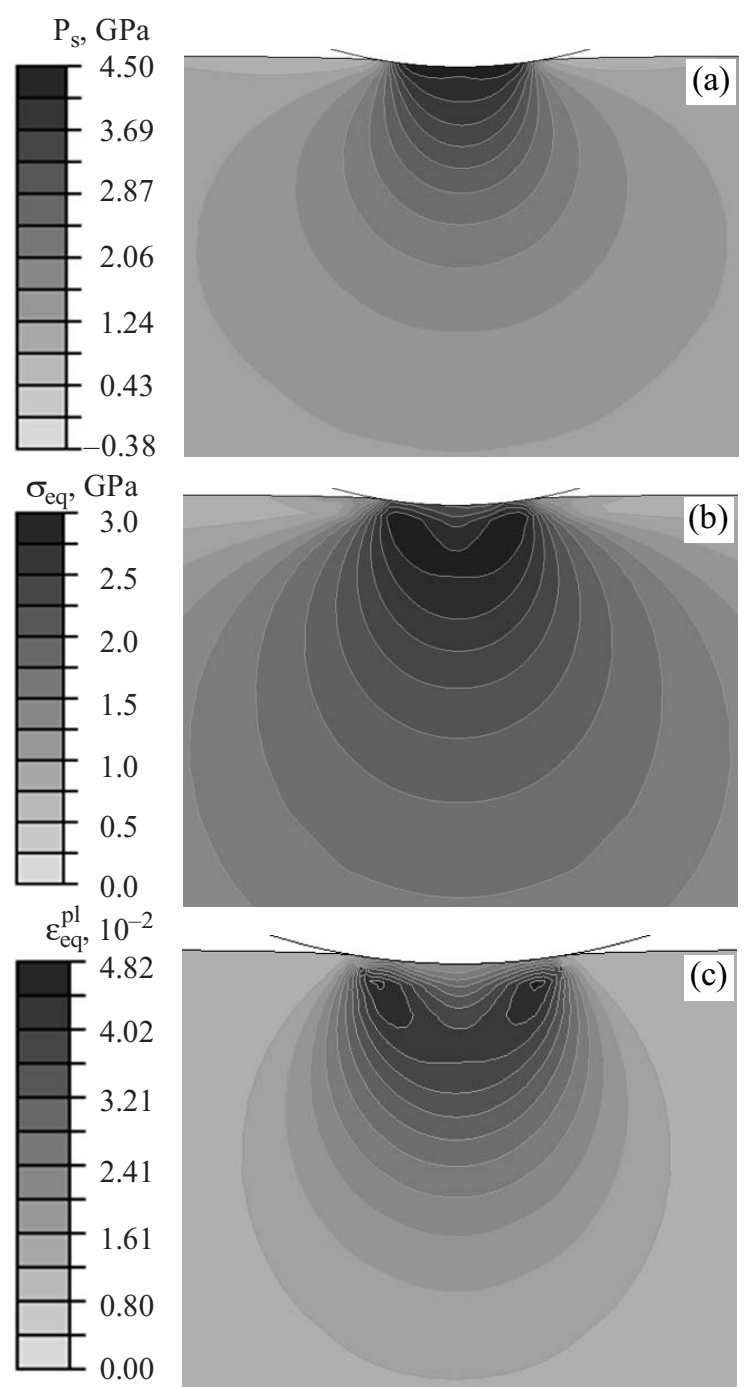

Fig. 4. Spatial distributions of the pressure (a), stress intensity (b), and accumulated plastic strain intensity (c) in the test specimen on completion of indentation. 


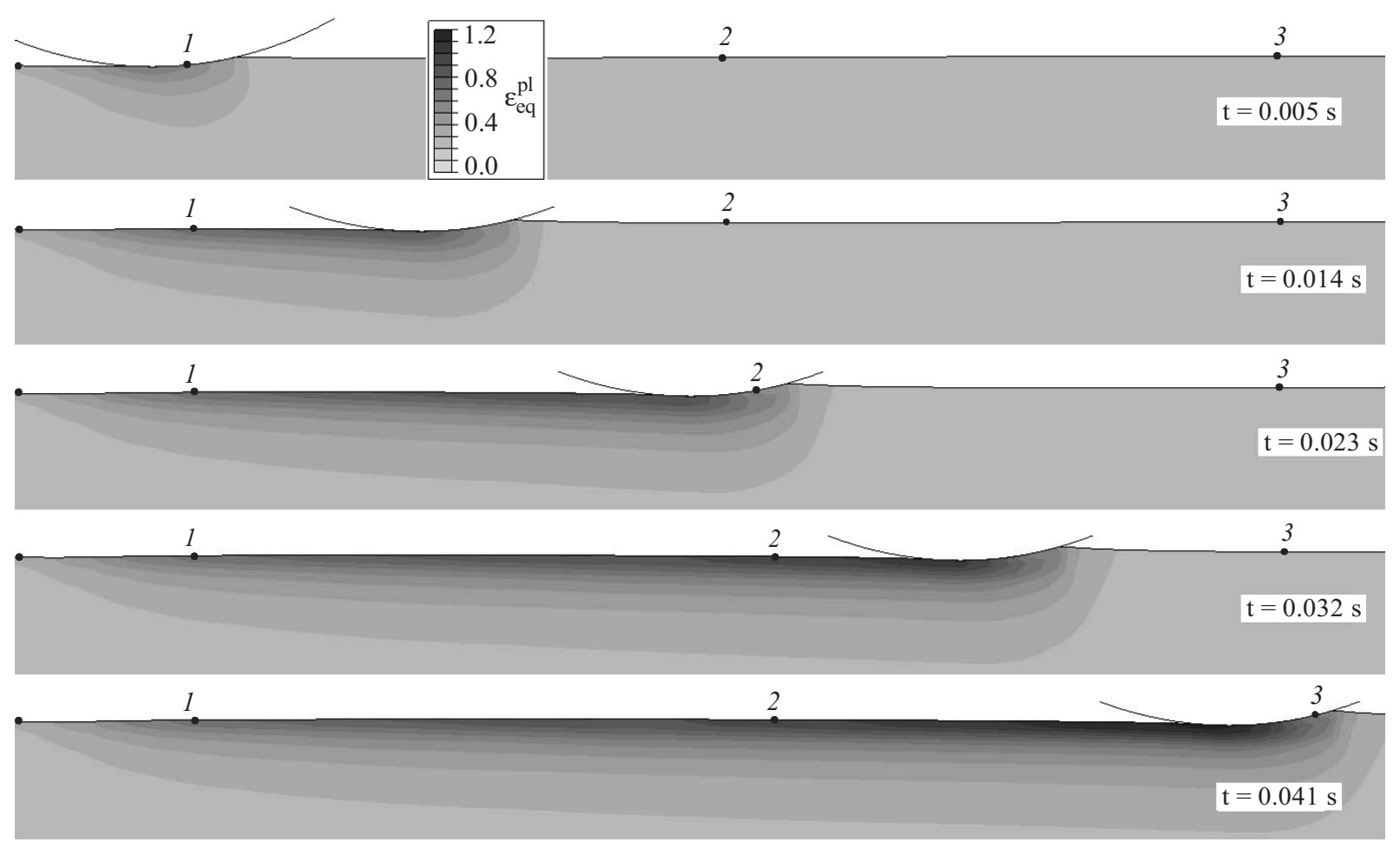

Fig. 5. Chronogram of the distribution of equivalent accumulated plastic strains. The layer thickness is $580 \mu \mathrm{m}$.

It is seen from the figure that directly beneath the indenter, there is a region of maximum uniform compressive stresses and shear stresses are found in a region a little bit deeper and sideways from the contact patch. The maximum tangential stresses are attained under extreme points of the contact patch from which bands of localized plastic deformation develop. It is in these zones that the largest number of structural defects should be expected in static indentation. Due to considerable linear hardening at small plastic strains (Fig. 3), the localization bands have rather "smeared" boundaries and the maximum strains in them are $\sim 5 \%$. Under static loading, the maximum plastic strains propagate to a depth of about $230 \mu \mathrm{m}$ and strains of $\sim 0.5 \%$ are found at a depth of up to $1400 \mu \mathrm{m}$.

\subsection{Plastic Strain Accumulation during Indenter Motion}

Next, we modeled loading of the specimen by an indenter moving with a constant velocity of $10 \mathrm{~m} / \mathrm{min}$ in the dynamic problem statement. Figure 5 shows a chronogram of the effective accumulated plastic strain distributions in the specimen loaded by a moving indenter. It is seen that ahead of the moving indenter, a bulge of edged material was formed. The plastic strains in the bulge are small, but the vertical displacements are maximal. It can also be traced on the chronogram that part of the material near the surface is entrained by the indenter; therefore, the position of points 2 and 3 by the end point in time is close to the right boundary of the cutout spatial domain.

Analysis of variations in accumulated plastic strains shows that under dynamic loading, high plastic strains are generated and accumulated beneath the moving indenter. So in a narrow near-surface layer of thickness

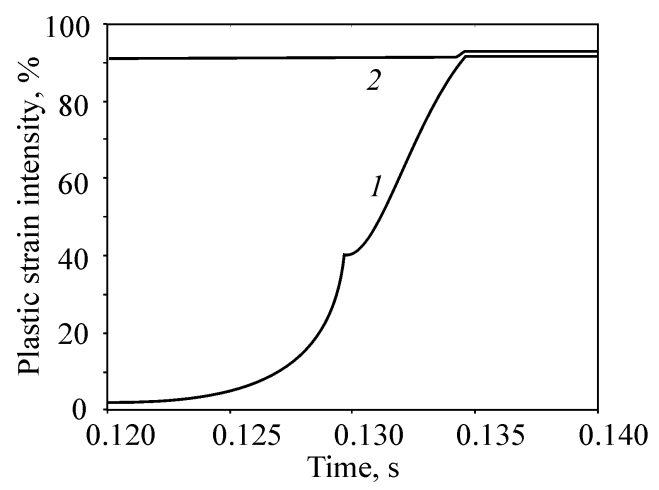

Fig. 6. Plastic strain accumulation in the middle of the specimen as a function of time and number of passes of the indenter (numerals near the curves). The time for each pass starts with zero. 


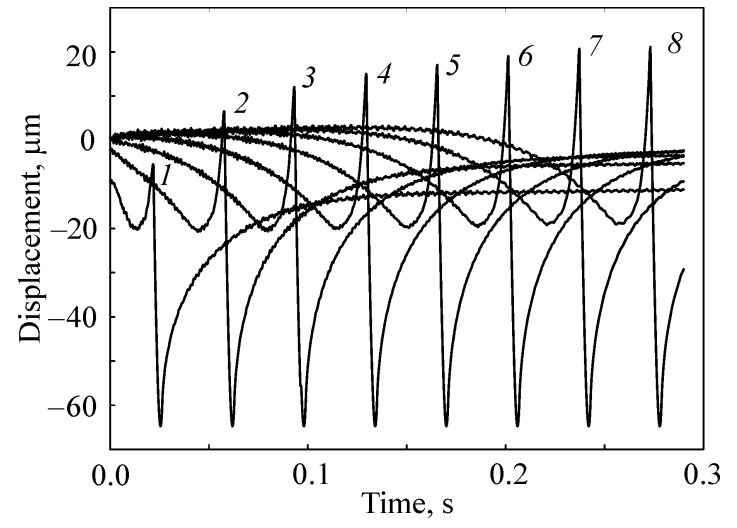

Fig. 7. Vertical displacements of surface points during the indenter motion. The numerals near the diagrams correspond to the numbers of equally spaced points, of which the first three are indicated in Fig. 5. The larger the number of a diagram, the farther a point is located from the initial indenter position.

$75 \mu \mathrm{m}$, they reach $\sim 100 \%$ and decrease in going deep into the material; at a depth of $\sim 500 \mu \mathrm{m}$, the plastic strains are $\sim 10 \%$.

In multiple passes of the indenter, plastic strain accumulation depends strongly on the form of the hardening curve used. If the hardening curve tends to a certain limiting value (to saturation), the strains increase nonmonotonically. If the hardening curve is approximated by a power function as in Fig. 3, considerable plastic strains are accumulated in the first pass and they remain further almost unchanged (Fig. 6). This means that a large number of passes can be inefficient for hardening the material and providing its other functional properties.

As one would expect, increasing the friction coefficient and the burnishing force increases the accumulated plastic strains in the thin surface layer subjected to nanostructuring burnishing [6].

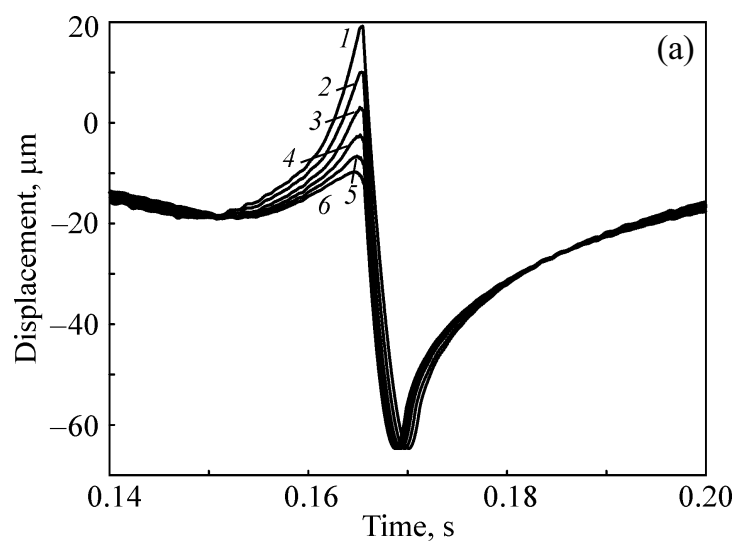

\subsection{Effect of the Friction Coefficient and Burnishing Force on the Height of a Bulge of Edged Material}

Figure 7 shows time dependences of vertical displacements on the specimen surface at certain points, part of which is marked by solid circles and numbers in Fig. 5 . The data were obtained at a burnishing force of $350 \mathrm{~N}$ and friction coefficient of 0.3 . Analysis of Fig. 7 suggests that even before arrival of the indenter, the surface points go down and then they lift up during the passage of the edged material bulge through them. As the indenter passes, the surface sinks and then lifts up again due to the presence of elastic strains. Diagrams 4 and 5 in Fig. 7 correspond to the boundary of a transition region (the region of stabilization of the edged material bulge) following which the displacements of surface points change but little. Point No. 5 is $2.7 \mathrm{~cm}$ away from the initial indenter position.

The effect of the friction coefficient and burnishing force on the height of the edged material bulge was analyzed from the diagrams of vertical displacements of the same point (No. 5) of the treated surface (Fig. 8). The bulge height was determined as a difference of the maximum lift in the bulge and the deepest sink for the point before arrival of the indenter.

It is seen that as the friction coefficient and the burnishing force are increased, the vertical displacements in the edged material bulge increase, but the elastic recovery of the indented material becomes lower. The maximum indentation (penetration) for one force is the same, but shifts farther from the beginning at high friction coefficients (the material is entrained by the indenter).

The effect of both burnishing parameters on the formation of the edged material bulge is represented in Fig. 9. It is seen that the increase in the height of the

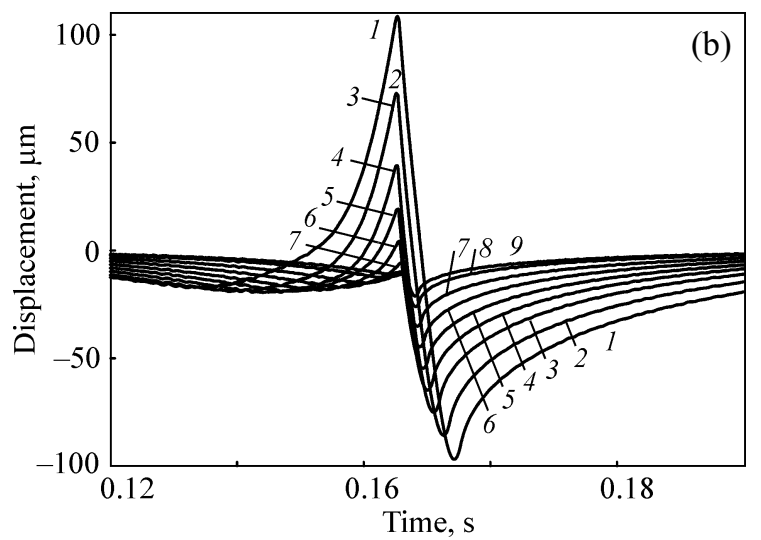

Fig. 8. Vertical displacements of the same point with time in the middle of the burnished surface at different friction coefficients $\mu=$ 0.35 (1), $0.3(2), 0.25(3), 0.2(4), 0.15(5), 0.1$ (6) and one burnishing force $P=350 \mathrm{~N}$ (a) and at different burnishing forces $P=500$ ( 1 ), 450 (2), $400(3), 350(4), 300(5), 250(6), 200(7), 150(8), 122.5(9)$ and one friction coefficient $\mu=0.35(b)$. 


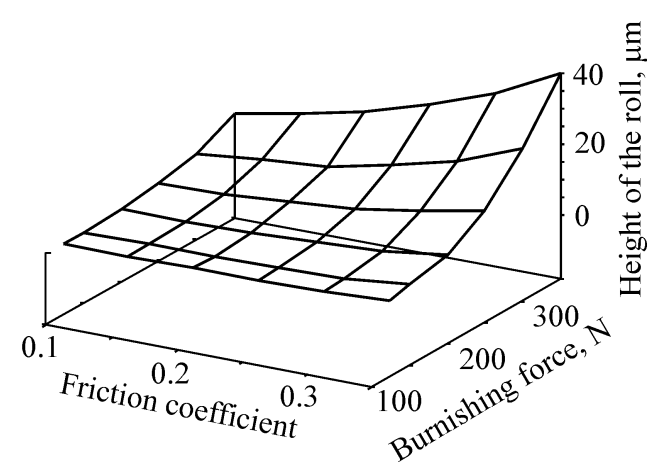

Fig. 9. Height of the bulge versus the friction coefficient and burnishing force.

edged material bulge with increasing the friction coefficient and the burnishing force obeys a nonlinear increasing dependence. Analysis of the calculation data also suggests that if the burnishing force is small, the bulge is hardly formed at all at friction coefficients of up to 0.35 . Additional calculations show that only at a very high friction coefficient $(\mu=0.5)$, the material is edged little.

\subsection{Stress Distribution at the Deformation Site}

Because the edged material bulge grows with an increase in both burnishing force and friction coefficient, let us analyze the stress distribution first for the case where the parameters are both high (the friction coefficient is 0.35 and the burnishing force is $350 \mathrm{~N}$ ), and then for that where they are both low (the friction coefficient is 0.07 and the burnishing force is $122.5 \mathrm{~N}$ ).

Figure 10a shows the indenter position, the deformed finite element grid, and the two-dimensional distribution of shear stresses $\sigma_{x y}$ in the computational domain. Figures $10 \mathrm{~b}$ and $10 \mathrm{c}$ show diagrams of the stress tensor components $\sigma_{x x}, \sigma_{y y}$ and $\sigma_{x y}$, and also the pressure $P_{\mathrm{s}}$ and the equivalent stresses $\sigma_{\text {eq }}$ along two lines indicated by markers in Fig. 10a. The abscissa is the distance from the edge of the marked line along the $x$ axis.

In analyzing the stresses in near-surface layers of the deformation site, it should be noted that lengthwise the indenter motion, the normal stresses $\sigma_{x x}$ upstream of the indenter (to its right) are negative, i.e., the material is compressed along the $x$ axis upstream of the indenter. At the deformation site, the situation gradually reverses and the stresses become positive, i.e., the material is stretched downstream of the indenter (entrained by the indenter due to friction). The material is compressed along the vertical axis only at the deformation site, and outside of the deformation site, the stress components $\sigma_{y y}$ are equal almost to zero. Slightly ahead of and directly at the deformation site, positive shear stresses operate. The uni- form compressive stresses begin to increase ahead of the bulge (the material is compressed due to arrival of the indenter), reach their maximum values behind the bulge (where the indenter presses the bulge), and further the pressure $P_{\mathrm{s}}$ throughout the deformation site remains compressive, giving way to tension downstream of the indenter due to friction-induced entrainment of the material surface layer there. Upstream of the indenter, shape changing also occurs. The maximum von Mises equivalent stresses, responsible for the shape changing, operate at the deformation site and remain there almost constant.
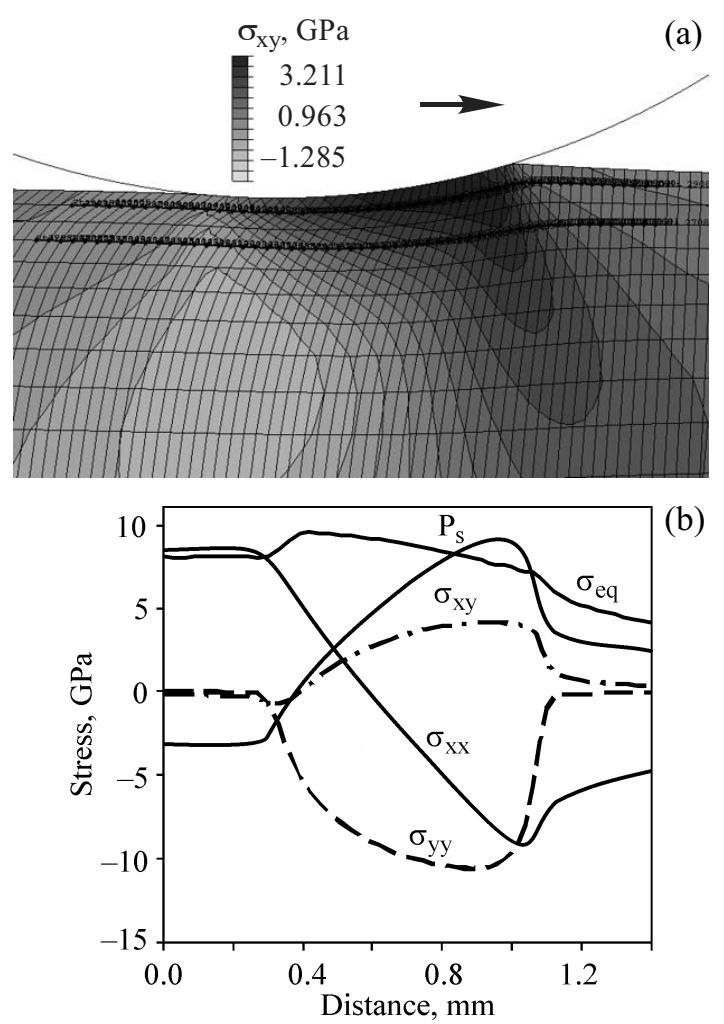

(b)

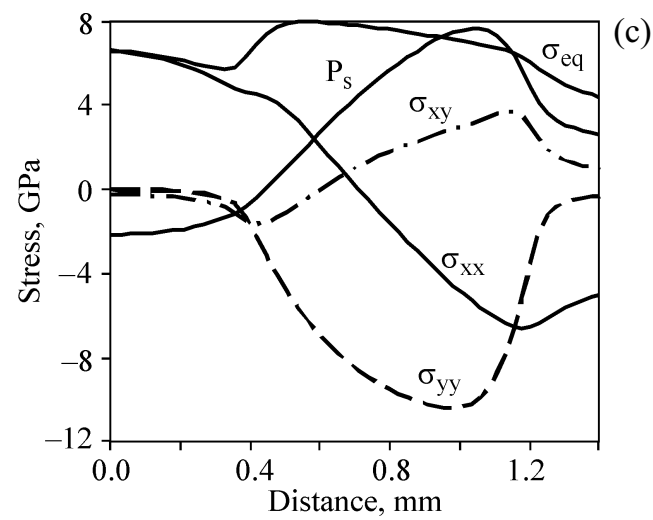

Fig. 10. Distribution of shear stresses at the deformation site (a) and different stress components along two lines at the surface (indicated by markers in (a)): $b$ - for the upper line, $c$ for the lower line with a large bulge of edged material. The direction of indenter motion is indicated by an arrow. 

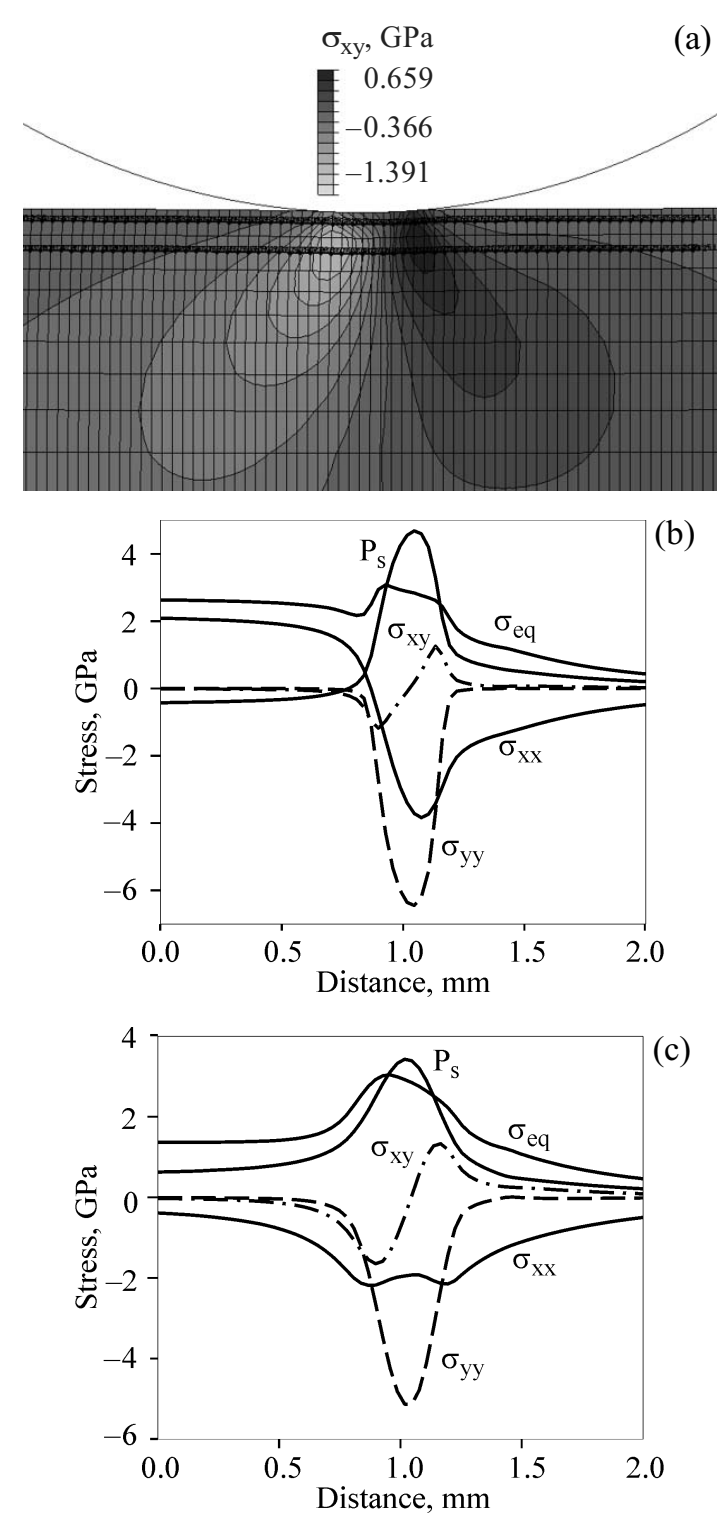

Fig. 11. Distribution of shear stresses at the deformation site (a) and different stress components along two lines at the surface (indicated by markers in (a)): b-for the upper line, cfor the lower line with a small bulge of edged material.

By and large, joint analysis of all stress components suggests that in the layers nearest to the surface, the stress state upstream of the deformation site is similar to axial compression and that downstream of this site is similar to uniaxial tension. At the deformation site, a complex stress state takes place.

In a deeper material layer, a different stress state downstream of the indenter is realized. It is seen that the lower line of markers in Fig. 10a crosses not only the region of positive shear stresses at the right (a wedge) but also the region of negative shear stresses at the left beneath the indenter. The patterns are precisely the same as those obtained earlier [22], and this suggests that vertical penetration of the indenter (without its surface motion) gives rise to two regions: a positive shear region and a negative shear region which are positioned symmetrically about the indenter axis. Motion of the indenter along the surface breaks down this symmetry and the shear stress distribution assumes the pattern shown in Fig. 10a.

The foregoing character of the stress distribution can explain the rotational shear deformation in a surface layer, because the indenter motion causes the uppermost surface region to experience alternate loads close to axial compression-tension and those deeper from the surface to experience alternate shear. In the upper surface layers, the plastic strains are thus higher than the plastic strain in the deeper material layers.

Another conclusion from the analysis of the stress distributions is that fracture of the surface layer is possible at high friction coefficients, because the normal tensile stresses can exceed the ultimate strength of the material.

Let us now consider the stress distribution at the deformation site when the bulge is hardly formed: the burnishing force is $P=122.5 \mathrm{~N}$ and the friction coefficient is 0.07 .

Figure 11 presents the same data as those in Fig. 10 but for the case when the bulge is very small. It is seen that the stress distribution in near-surface layers qualitatively fits that for a large bulge. A difference is found only in the shear stresses $\sigma_{x y}$, whose distribution is closer to asymmetric about the indenter vertex. In deeper layers, a qualitative difference is found in the normal stress distribution $\sigma_{x x}$; in this case, no transition from compression upstream of the indenter to tension downstream of it is observed. All layers experience compression.

By and large, the stress distribution patterns obtained in our calculations agree qualitatively with the data of numerical experiments [21]. The main differences of our results from those of [22] are the absence of negative shear stresses in the uppermost layers with a large bulge and the change of sign of longitudinal normal stresses in the uppermost layers with a small bulge and in deeper layers with a large bulge. However, these features are quite explainable in the context of the dynamic problem statement and joint action of burnishing forces and friction, and can give an insight into the formation mechanism of a nanostructured layer.

\section{DISCUSSION AND CONCLUDING REMARKS}

The most important result of the study is disclosure of the conditions conductive to the formation of a nanostructured near-surface layer in burnishing. In particular, 

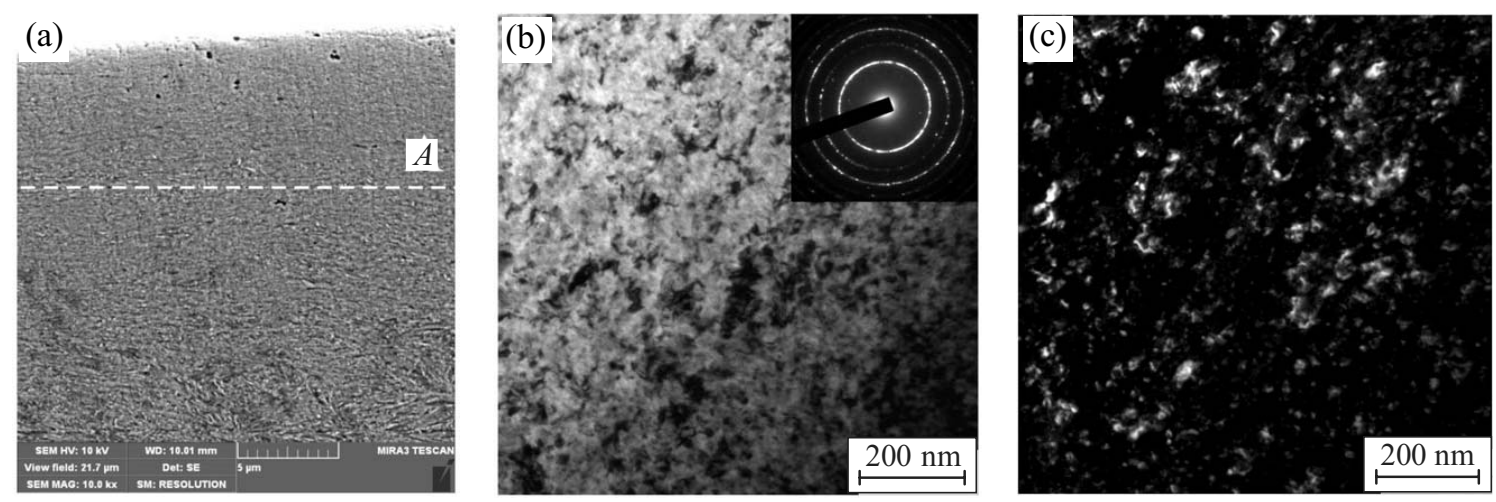

Fig. 12. Scanning and transmission electron microscopy of a thin surface layer of carburized steel 20X subjected to nanostructuring burnishing by a tool with a dynamic stabilization unit on a turn-mill center: $A$-nanostructured layer boundary.

analysis of the numerical simulation results shows that motion of the indenter along the burnished surface gives rise to a complex stress-strain state in the material region near the indenter and its dynamics reveals an alternating character. The material near the free surface upstream of the indenter is under compressive stresses, and after passage of the indenter, the stresses reverse, i.e., a region of tensile stresses arises. At a larger depth, this shows up in the change of sign of shear stresses. Multiple passes of the indenter along the surface of a treated part during its rotation on a machine create cyclic alternate loads on near-surface layers of the material. The available methods of severe plastic deformation resulting in nanostructured bulk metal materials are characterized by large degrees of shear strains with a variable stress-strain state $[31,32]$. The probability of these conditions to occur in frictional hardening of steels is also pointed out elsewhere $[26,27,33]$.

The experiments [6] confirm the formation of nanostructured layers in burnishing. Transmission electron microscopy shows that in a thin surface layer of carburized steel 20X subjected to nanostructuring burnishing by an indenter with a high friction coefficient $\left(K_{\mathrm{f}}=\right.$ 0.35 ), a homogeneous ultrafine-grained structure with $\alpha$ martensite crystallite sizes of $20-50 \mathrm{~nm}$ is formed (Figs. 12b and 12c). The electron diffraction patterns in the form of continuous Debye rings suggest that individual fragments are misoriented at angles ranging to several tens of degrees. The rotational plasticity in nanostructuring surface burnishing of steel $20 \mathrm{X}$ ensures the formation of nanocrystalline structures in a surface layer at a depth of up to 5-7 $\mu \mathrm{m}$ (Fig. 12a).

Thus, the distribution of accumulated plastic strains and stresses in the upper layers of the burnished surface shows that a narrow near-surface layer undergoes certain peculiar severe plastic deformation. It is this action on the surface layer material that can provide the formation of a nanograined structure in burnishing. The results of numerical simulation demonstrate that the depth of a surface layer subjected to external action is determined by the process parameters: the burnishing force and the friction coefficient. Increasing the friction coefficient increases the shear stresses and the accumulated plastic strains, and this assists the formation of nanostructural states. On the other hand, at very high friction coefficients, the burnishing mode can change with break of the contact and fracture of the surface layer material.

In conclusion, it should be noted that the results of the study in the quasistatic problem statement agree well with the data obtained by other authors, and this points to correctness of the used physical model of the process. The results obtained in the dynamic problem statement give qualitatively new information on the behavioral peculiarities of material under loading. They make it possible to analyze the stress and strain distributions in a loaded material under conditions close to those of surface burnishing of metal materials and to elucidate the effect of the burnishing force, the indenter sizes and shape, and the friction coefficient of a contact pair on the depth and properties of a nanostructured layer. Analysis of the simulation results has allowed us to determine the main parameters and the treatment modes that are capable of providing the mechanisms responsible for the formation of a nanostructured layer.

The main results of the study can be formulated as follows.

The contribution of longitudinal normal and shear stress components as parameters that provide nanostructuring of material surface layers of differing thickness has been revealed. 
It is shown that increasing the friction coefficient in burnishing increases the shear strains in a surface layer. The in-depth change in the amplitude and sign of these strains provides rotational-shear mechanisms of nanostructuring burnishing with high friction.

It is found that the increase in accumulated plastic strains in a surface layer under multiple actions is purely nonuniform.

It is demonstrated that nanostructuring can be controlled by choosing an appropriate ratio between the specific pressure at the indenter - surface contact patch and the friction coefficient.

The constraint factor for nanostructuring burnishing is the formation of dangerous tensile-compressive stresses at the deformation site and their ability to cause fracture.

The next step in analyzing the structural changes in a thin surface layer is in additional studies, including numerical simulation on a lower scale.

The work was partially support by project Nos. 09-T1-1002 and 13.3 under program of the Department of Energy, Engineering, Mechanics, and Control Processes RAS No. 13, integration project of SB RAS No. 127, and RFBR grant No. 11-08-01025-a.

\section{REFERENCES}

1. Korzynski, M. and Pacana, A., Centreless Burnishing and Influence of its Parameters on Machining Effects, J. Mater. Process. Tech., 2010, vol. 210, no. 9, pp. 1217-1223.

2. Sharman, A.R.C., Aspinwall, D.K., Dewew, R.C., and Bowen, P., Workpiece Surface Integrity Considerations when Finish Turning Gamma Titanium Aluminide, Wear, 2000, vol. 294, pp. 473-481.

3. Kuznetsov, V.P., Gorgots, V.G., Gubanov, V.F., Skhirtladze, A.G., Surface Control Management based on Burnishing Processes, Kurgan: Izd. Kurgan Gos. Univer., 2005.

4. Kuznetsov, V.P., Nonlinear Dynamic Simulation of Tools Parameters of Elastic Burnishing, Mashinostr., Inzh. Obrazov., 2009, vol. 20, no. 3, pp. 18-26.

5. Kuznetsov, V.P., Makarov, A.V., Savrai, R.A., Pozdeeva, N.A., Malygina, I.Yu., and Kiryakov, A.E., High-Quality Surfacing of Corrosion-Resistance Parts by Burnishing using a Turn/Mill Machine, Uprochn. Tekhnol. Pokryt., 2011, no. 8, pp. 3-15.

6. Kuznetsov, V.P., Makarov, A.V., Pozdeeva, N.A., Savrai, R.A., Yurovskii, A.S., Malygina, I.Yu., and Kiryakov, A.E., Enhancement in Strength, Heat and Wear Resistance in Parts of Carburized 20X Steel by Nanostructuring Burnishing using Turn/Mill Machines, Uprochn. Tekhnol. Pokryt., 2011, no. 9, pp. 3-13.

7. Hamadache, H., Laouar, L., Zeghib, N.E., and Chaoui, K. Characteristics of Rb40 Steel Superficial Layer under Ball and Roller Burnishing, J. Mater. Process. Tech., 2006, vol. 180, pp. $130-136$.

8. Loh, N.H., Tam, S.C., and Miyazawa, S., Surface Gardening by Ball Burnishing, Proc. Eng., 1990, vol. 23, no. 6, pp. 413-417.

9. Michael, P.C., Saka, N., and Rabinowicz, E., Burnishing and Adhesive Wear of an Electrically Conductive Polyester-Carbon Film, Wear, 1989, vol. 132, no. 2, pp. 265-285.

10. Hassan, A.M., The Effect of Ball and Roller Burnishing on the Surface Roughness of Some Non-ferrous Metals, $J$. Mater. Process. Tech., 1997, pp. 385-391.

11. Shiou, F.J. and Hsu, C.C., Surface Finishing of Hardened and Tempered Stainless Tool Steel Using Sequential Ball Grinding, Ball Burnishing and Ball Polishing Processes on a Machining Centre, J. Mater. Process. Tech., 2008, vol. 205, no. 1-3, pp. 249-258.

12. Korzynski, M., A Model of Smoothing Slide Ball-Burnishing and an Analysis of the Parameter Interaction, J. Mater. Process. Tech., 2009, vol. 209, no. 1, pp. 625-633.

13. Shiou, F.J. and Chuang, C.H., Precision Surface Finish of the Mold Steel PDS5 Using an Innovative Ball Burnishing Tool Embedded with a Load Cell, Precis. Eng., 2010, vol. 34 , no. 1, pp. 76-84.

14. Luo, H., Liu, J., Wang, L., and Zhong, Q., Study of the Mechanism of the Burnishing Process with Cylindrical Polycrystalline Diamond Tools, J. Mater. Process. Tech., 2006, vol. 180 , no. 1-3, pp. 9-16.

15. Korzynski, M., Lubas, J., Swirad, S., and Dudek, K., Surface Layer Characteristics due to Slide Diamond Burnishing with a Cylindrical-Ended Tool, J. Mater. Process. Tech., 2011, vol. 211, pp. 84-94.

16. Schneider, G., Jr., Cutting Tool Applications, Englewood Cliffs, N.J.: Prentice-Hall Publication, 2002, Chapter 1, pp. 2-65.

17. Odintsov, V.G., Finishing Treatment by Diamond Burnishing and Vibroburnishibg, Moscow: Mashinostroenie, 1981.

18. Kuznetsov, V.P., Simulation of the Effect Technological Parameters on Vibration Resistance in Surface Burnishing, Metalloobrabotka, 2010, vol. 55, no. 1, pp. 7-15.

19. Pshibylskii, V., Technology of Surface Plastic Treatment, Moscow: Metallurgiya, 1991.

20. Kucheryaev, B.V., Mechanics of Continuum. Theoretical Foundations of Composite Plastic Working, Moscow: MISIS, 2000.

21. Smelyanskii, V.M., Mechanics of Material Strengthening by Surface Plastic Deformation, Moscow: Mashinostroenie, 2002.

22. Sartkulvanich, P., Altan, T., Jasso, F., and Rodriguez, C., Finite Element Modeling of Hard Roller Burnishing: An Analysis on the Effects of Process Parameters upon Surface Finish and Residual Stresses, J. Manuf. Sci. Eng., 2007, vol. 129, iss. 4, p. 705.

23. Yen, Y.C., Sartkulvanich, P., and Altan, T., Finite Element Modeling of Roller Burnishing Process, CIRP Annals Manuf. Technol., 2005, vol. 54, iss. 1, pp. 237-240. 
24. Courtin, S., Henaff-Gardin, C., and Bezine, G., Finite Element Simulation of Roller Burnishing in Crankshafts, Transactions on Engineering Sciences, 2003, vol. 39, pp. 333-342.

25. Klocke, F., Bäcker, V., Wegner, H., and Zimmermann, M., Finite Element Analysis of the Roller Burnishing Process for Fatigue Resistance Increase of Engine Components, Proc. Inst. Mech. Engrs, Part B: J. Engng Manuf., 2011, vol. 225, no. 1, pp. 2-11.

26. Makarov, A.V., Savrai, R.A., Pozdejeva, N.A., Smirnov, S.V., Vichuzhanin, D.I., Korshunov, L.G., and Malygina, I.Yu., Effect of Hardening Friction Treatment with Hard-Alloy Indenter on Microstructure, Mechanical Properties, and Deformation and Fracture Features of Constructional Steel under Static and Cyclic Tension, Surf. Coat. Tech., 2010, vol. 205, pp. 841-852.

27. Vichuzhanin, D.I., Makarov, A.V., Smirnov, S.V., Pozdeeva, N.A., and Malygina I.Yu., Stress-Strain State and Damage at Friction Strengthening of Steel Plane Surface by Cylindrical Indenters, Problem. Mashinoved. Nadezhn. Mashin, 2011.
28. Panin, V.E. and Egorushkin, V.E., Nanostructural States in Solids, Phys. Met. Metallogr., 2010, vol. 110, no. 5, pp. 464-473.

29. Panin, V.E. and Panin, A.V., Effect of the Surface Layer in a Solid under Deformation, Phys. Mesomech., 2005, vol. 8, no. 5-6, pp. 7-14.

30. Konovalov, D.A., Smirnov, S.V., and Konovalov, A.V., Determination of Metal Strain-Hardening Curves from Conical Indenter Impression Results, Russ. J. Nondestr. Test., 2008, no. 12, pp. 55-63.

31. Valiev, R.Z. and Alexandrov, I.V., Nanostructured Materials Obtained by Severe Plastic Deformation, Moscow: Logos, 2000.

32. Valiev, R.Z., Estrin, Yu., Horita, Z., Langdon, T.G., Zehetbauer, M.J., and Zhu, Yu.T., Producing Bulk UltrafineGrained Materials by Severe Plastic Deformation, JOM, 2006, vol. 4, pp. 33-39.

33. Kragelskii, I.V., Friction and Wear, Washington: Butterworths, 1965. 